\title{
Electroluminescence at silicon band gap energy from mechanically pressed indium-tin-oxide/Si contact
}

\author{
Ching-Fuh Lin, ${ }^{\text {a) }}$ Miin-Jang Chen, Shu-Wei Chang, Peng-Fei Chung, Eih-Zhe Liang, \\ Ting-Wien Su, and C. W. Liu \\ Department of Electrical Engineering and Graduate Institute of Electro-Optical Engineering, \\ National Taiwan University, Taipei, Taiwan, Republic of China
}

(Received 21 November 2000; accepted for publication 5 February 2001)

\begin{abstract}
Room temperature electroluminescence (EL) corresponding to Si band gap energy is observed from mechanically pressed indium-tin-oxide (ITO)/Si contact. The intensity of luminescence is pressure dependent and highly related to the current-voltage characteristics. Increasing pressure simultaneously reduces the rectification property and the luminescence. The physical reason for EL is attributed to the formation of an air gap between the ITO and the Si substrate. The role of the air gap is similar to the oxide layer in the metal-oxide-semiconductor structure. The influence of surface quality of the $\mathrm{Si}$ substrate on the luminescence spectrum is also studied, and found to be significant. (C) 2001 American Institute of Physics. [DOI: 10.1063/1.1359138]
\end{abstract}

Silicon has been popularly used by the integrated-circuit industry. However, its applications in areas of optics and optoelectronics are much less due to the indirect band gap nature that makes it hardly emit light. To monolithically integrate electronics and optoelectronics well, many efforts had been devoted to converting silicon to a light-emitting material or causing luminescence on silicon substrates. They include porous-silicon-based devices, ${ }^{1-3}$ nanocrystalline $\mathrm{Si}^{4,5}$ $\mathrm{Si}^{+}$-implanted $\mathrm{SiO}_{2},{ }^{6,7}$ Er-doped $\mathrm{Si}^{8,9}$ deposition of polymer on $\mathrm{Si}$ substrates, ${ }^{10}$ growth of $\mathrm{GaN}$ on $\mathrm{Si}$ substrates, ${ }^{11,12}$ metal-oxide-semiconductor (MOS) structures with native oxide, ${ }^{13,14}$ and so on. Among them, the obstacle of indirect band gap nature of $\mathrm{Si}$ was never overcome, so no specific emission at Si band gap energy was observed. Recently, we discovered that MOS structures on $\mathrm{Si}$ with the oxide grown by the well-controlled rapid thermal oxidation (RTO) could have the electroluminescence (EL) corresponding to Si band gap energy. ${ }^{15,16}$ In this work, we report the further discovery that the insulation layer does not have to be the oxide grown by RTO. Room temperature EL corresponding to Si band gap energy could also occur from mechanically pressed indium-tin-oxide (ITO)/Si contact. The pressure-dependent current-voltage $(I-V)$ characteristics and the luminescence are investigated. The influence of the Si surface quality on the luminescence is also examined.

The experimental setup is schematically shown in Fig. 1. A piece of glass with one side deposited with ITO was used as the base to support the structure. The native oxide on the polished side of the Si substrate was removed by the bufferoxide-etchant (BOE). Aluminum metal was deposited on the other side of the Si substrate for electrical contact. Then the Si substrate was placed on top of the glass with its polished surface facing the ITO surface of the glass. Two wires were connected to the metal side of Si and the ITO on the glass, respectively. Either forward- or reverse-biased voltages were applied to the setup through these two wires. Here, the forward or reverse bias is defined according to the conventional

${ }^{a)}$ Electronic mail: cflin@cc.ee.ntu.edu.tw ways. ${ }^{15}$ On top of the Si substrate, the pressure was applied through a micrometer that was tightly held to the glass, so the pressure could be varied by turning the micrometer. A stainless-steel ball was used to press down the $\mathrm{Si}$ wafer in order to maintain a nearly uniform distance between the $\mathrm{Si}$ surface of the $2 \times 2 \mathrm{~mm}^{2}$ area and the ITO surface.

Without the applied pressure, the Si surface has no close contact with the ITO surface, so no current flows through the structure. Under a certain pressure, current appears when the structure is under forward bias. Increasing pressure causes both forward- and reverse-biased currents to gradually increase. Figure 2 shows the pressure-dependent $I-V$ characteristics for the experiment using $n$-type $\mathrm{Si}$. The rectification characteristics are obvious, but are reduced as the pressure increases. Both $p$-type and $n$-type Si had been experimented on and showed similar behaviors, except with different voltage polarities.

The mechanically pressed ITO/Si contact behaves like a metal-insulator-semiconductor (MIS) tunneling diode because an air gap is formed between the $\mathrm{Si}$ substrate and the ITO surface to serve as the insulation layer. When the air gap is narrow enough, carriers could quantum-mechanically tunnel through. Under forward bias, EL could be measured even with a current density as low as $0.6 \mathrm{~A} / \mathrm{cm}^{2}$. Figure 3 shows the EL spectra at different injection currents. The spectral shape of the band edge emission is similar to that measured from the MOS structure with the RTO grown oxide. ${ }^{15,16}$ No luminescence is observed when the structure is reversebiased.

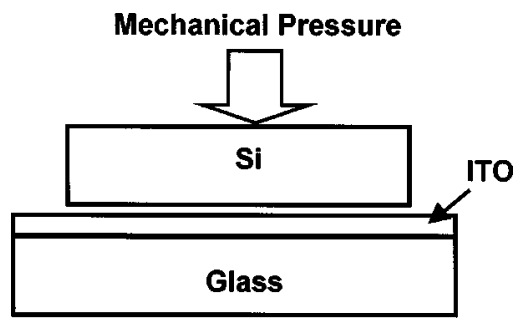

FIG. 1. Schematic setup of the mechanically pressed ITO/Si contact. 


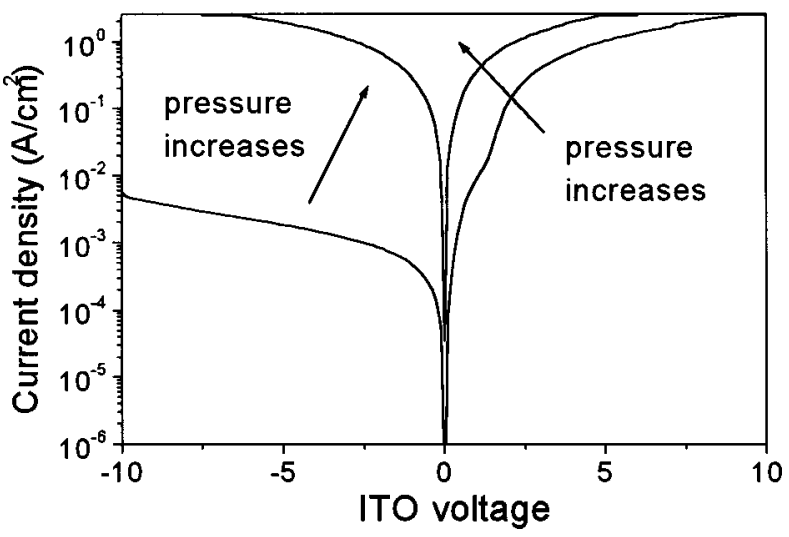

FIG. 2. Pressure-dependent $I-V$ characteristics with $n$-type Si used in the experiment.

From the $I-V$ characteristics and the emission spectrum, the physical reason for the EL should be similar to the MOS with the RTO grown oxide. ${ }^{15,16}$ The formation of a potential well at the Si/insulator interface is crucial for the EL. For $n$-type $\mathrm{Si}$, a huge amount of electrons accumulate in the potential well when the structure is under forward bias. The tunneling holes also simultaneously appear in the well region for enhanced radiative recombination. For $p$-type $\mathrm{Si}$, similar behaviors also occur, except that the roles of electron and hole are switched. The carrier confinement in the potential well causes the wave function to be localized, and so creates the spread of the lattice momentum in the reciprocal lattice. Plus, with the band-filling effect other mechanisms, such as interface roughness, phonon, and impurity scattering, could then more easily provide enough momentum for the radiative recombination.

The EL emission strength also depends on the applied pressure, as shown in Fig. 4. Under the constant forward current the EL decreases with the pressure, and eventually vanishes before over-pressure breaks the glass. The large pressure also reduces the $I-V$ rectification characteristics. The reason is because the ITO surface has more direct contact with Si surface under great pressure. The direct contact of ITO with $\mathrm{Si}$ is nearly ohmic and causes no electrons (or holes) to accumulate near the $\mathrm{Si}$ /insulator interface, reducing EL. Si substrates with the native oxide remained on the surface have also been experimented on in the setup. The experiment shows that the EL efficiency also decreases with the applied pressure, but not to zero. The native oxide pre-

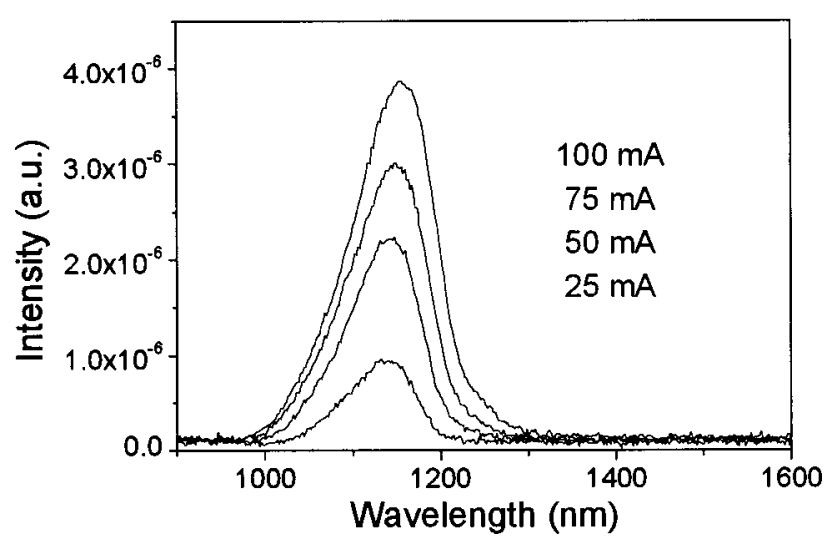

FIG. 3. EL spectra from the mechanically pressed MIS structure.

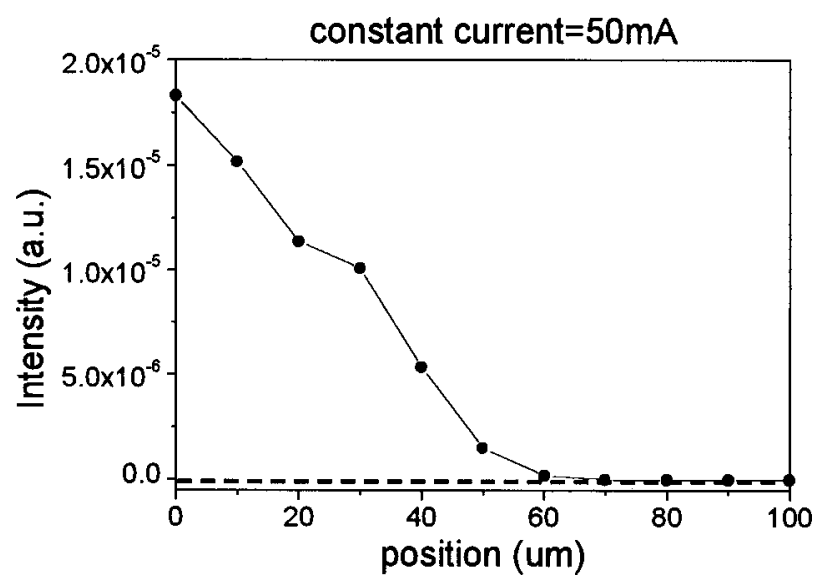

FIG. 4. EL emission strength vs micrometer reading (applied pressure).

vents the MIS structure from having direct contact of ITO and Si everywhere, so electrons or holes could still accumulate at the oxide/Si interface in some area, for the generation of EL.

The EL spectrum is discovered to be highly dependent on the surface quality of the Si wafer. To change the surface quality, the polished side of the Si substrate is lapped using sand paper (No. 400). This Si substrate is then put in the setup shown in Fig. 1 with the lapped side facing the ITO surface of the glass. Such a MIS structure also exhibits EL, but has a very broad spectrum. Figure 5(a) shows the measured spectrum from 1.0 to $1.7 \mu \mathrm{m}$, limited by the spectral range of the InGaAs detector. Because lapping creates many surface states with their energies situating in the band gap, electrons in the conduction band could transit to those sur-
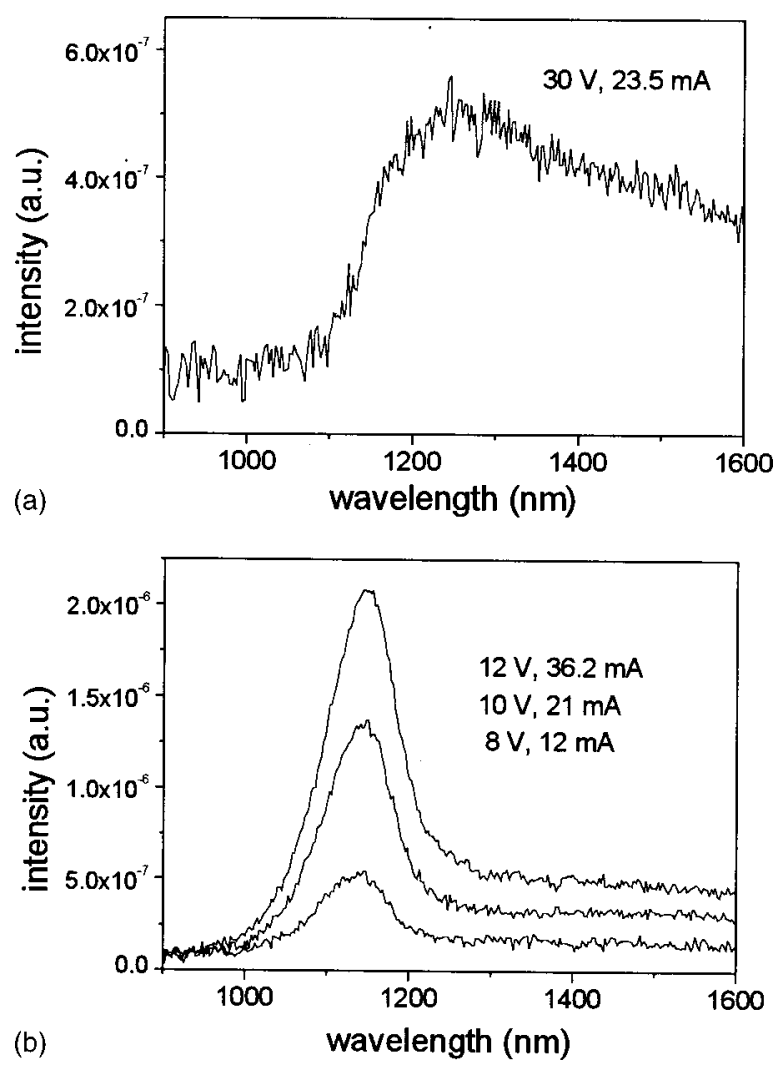

FIG. 5. EL spectra (a) with lapped Si surface. (b) Chemically repolished Si surface. 
face states. The measured EL indicates that such transition could be radiative. Such transition also reduces the emission at the band gap energy. As the lapped Si surface is chemically repolished, the EL again has a major emission at the band gap energy, accompanied with relatively small EL at other energies, as shown in Fig. 5(b). This indicates that the later chemical polish reduces the surface states, but does not completely remove them. This experiment reveals that the electron-hole radiative recombination and the transition through surface states are competing processes.

The experiment also shows that the simple setup shown in Fig. 1 could be used for surface-quality inspection. Similar to the photoexcitation in photoluminescence experiments, the MIS structure provides the mechanism for up-lifting electrons to high-energy states by quantum tunneling. The tunneled hot electrons then relax to lower energy states by emitting photons with corresponding energies. The resulting EL spectrum could be used for the interpretation of surface ingredients.

In conclusion, room temperature EL at $\mathrm{Si}$ band gap energy is observed from the mechanically pressed ITO/Si contact. The $I-V$ curve of the mechanically pressed MIS structure and the EL intensity are pressure-dependent. Increasing pressure simultaneously reduces the $I-V$ rectification characteristics and the luminescence. The formation of an air gap between the ITO surface and the Si substrate is crucial for the EL. The role of the air gap is similar to the RTO grown oxide in the MOS structure. The surface quality of Si substrate is also experimentally found to have significant influences on the luminescence spectrum, because of the compe- tition between the band edge emission and the transition through surface states.

This work is supported in part by the National Science Council, Taipei, Taiwan, R.O.C. under Contract Nos. 892215-E-002-059 and 89-2112-M-002-076.

${ }^{1}$ L. T. Canham, Appl. Phys. Lett. 57, 1046 (1990).

${ }^{2}$ N. Koshida and H. Koyama, Appl. Phys. Lett. 60, 347 (1992).

${ }^{3}$ S. Lazarouk, P. Jaguiro, S. Katsouba, G. Masini, S. La Monica, G. Maiello, and F. Ferrari, Appl. Phys. Lett. 68, 2108 (1996).

${ }^{4}$ S. Schuppler, S. L. Friedman, M. A. Marcus, D. L. Adler, Y.-H. Xie, F. M. Ross, Y. J. Chabal, T. D. Harris, L. E. Brus, W. L. Brown, E. E. Chaban, P. F. Szajowski, S. B. Christman, and P. H. Citrin, Phys. Rev. B 52, 4910 (1995).

${ }^{5}$ D. B. Geohegan, A. A. Puretzky, G. Duscher, and S. J. Pennycook, Appl. Phys. Lett. 73, 438 (1998).

${ }^{6}$ L. S. Liao, X.-M. Bao, X.-Q. Zheng, N.-S. Li, and N.-B. Min, Appl. Phys. Lett. 68, 850 (1996).

${ }^{7}$ W. Skorupa, R. A. Yankov, I. E. Tyschenko, H. Frob, T. Bohme, and K. Leo, Appl. Phys. Lett. 68, 2410 (1996).

${ }^{8}$ G. Franzo, F. Priolo, S. Coffa, A. Polman, and A. Carnera, Appl. Phys. Lett. 64, 2235 (1994).

${ }^{9}$ M. Matsuoka and S. Tohno, Appl. Phys. Lett. 71, 96 (1997).

${ }^{10}$ D. R. Baigent, R. N. Marks, N. C. Greenham, R. H. Friend, S. C. Moratti, and A. B. Holmes, Appl. Phys. Lett. 65, 2636 (1994).

${ }^{11}$ R. Birkhahn and A. J. Steckl, Appl. Phys. Lett. 73, 1700 (1998).

${ }^{12}$ M. Garter, J. Scofield, R. Birkhahn, and A. J. Steckl, Appl. Phys. Lett. 74, 182 (1999).

${ }^{13}$ Y. Q. Wang, T. P. Zhao, J. Liu, and G. G. Qin, Appl. Phys. Lett. 74, 3815 (1999).

${ }^{14}$ J. Yuan and D. Haneman, J. Appl. Phys. 86, 2358 (1999).

${ }^{15}$ C.-F. Lin, C. W. Liu, M.-J. Chen, M. H. Lee, and I. C. Lin, J. Appl. Phys. 87, 8793 (2000).

${ }^{16}$ C. W. Liu, M.-J. Chen, I. C. Lin, M. H. Lee, and C.-F. Lin, Appl. Phys. Lett. 77, 1111 (2000). 